Supporting Information for

\title{
The stages that lead to drop depinning and onset of motion
}

Akash K Jena ${ }^{1}$, Yagna Valkya Reddy Bhimavarapu ${ }^{1}$, Sirui Tang ${ }^{1}, \mathrm{Jie} \mathrm{Liu}^{1}$, Ratul Das ${ }^{2}$, Semih $\mathrm{Gulec}^{1}$, Appu Vinod $^{1}$, Chun-Wei Yao ${ }^{3}$, Tianxing Cai ${ }^{1}$, Rafael Tadmor ${ }^{* 4}$

*email: tadmorr@post.bgu.ac.il

1. Dan F. Smith Dept. of Chemical \& Biomolecular Engineering, Lamar University, P. O. Box 10053, Beaumont TX 77710

2. Innovation and New Technology, ACWA Power, KAUST ACWA Power Center of Excellence, Thuwal, Makkah, Saudi Arabia 23955

3. Department of Mechanical Engineering, Lamar University, P.O. Box 10053, Beaumont TX 77710

4. Department of Mechanical Engineering, Ben Gurion University, Beer Sheva, Israel 84105

Table of Contents

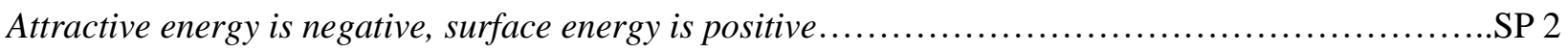

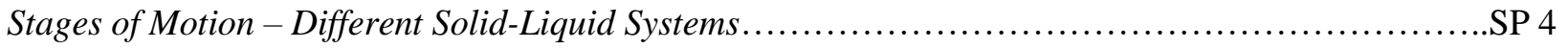




\section{SC1: Attractive energy is negative, surface energy is positive}

Two terms that are used throughout the text are attractive energy and surface energy (the latter is often called surface tension in relation to its liquid-vapor form). Below we explain that attractive energy is a negative property and surface energy is a positive property, yet the maxima and minima of these two properties coincide.

\section{Attractive energy is negative}

By "attractive energy" we refer to the energy associated with the intermolecular interaction between two adjacent layers such as solid-liquid, solid-air, and liquid-air. Since the layers are adjacent, a dipole force (such as London-van der Waals interaction) results in an attraction, i.e., a negative energy*. Stronger attraction results in a lower energy (deeper negative energy well which coincides with our intuition that emanates from gravitational potential energy with a lower value for a valley and higher value for a mountain). We conclude that the energy of interaction between two adjacent layers is attractive and is represented by a negative value.

* The only case in which the interaction of adjacent layers is positive is when the surfaces are charged and the counterions cannot screen the interaction for various reasons. However, we consider dipole forces for which attraction is the only option.

\section{Surface energy is positive}

Surface energy represents the work that one needs to invest to create a surface. For example, the surface energy of a solid with air, $\gamma_{S}$, is the work required to split a solid continuum and separate its two halves from contact to infinity as demonstrated in Figure S1.

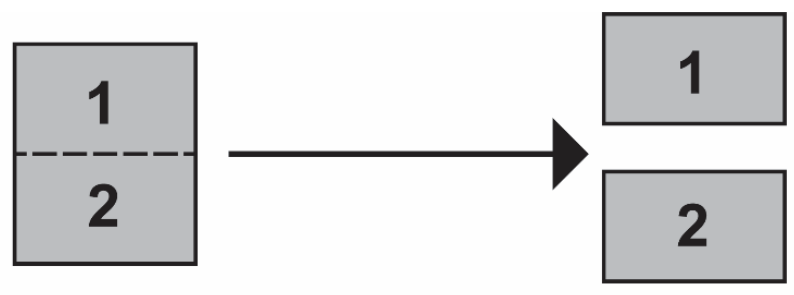

\section{a)}

b)

Figure S1: (a) a solid continuum in which an imaginary border is drawn between two conceptual halves; (b) The two halves after being separated.

Since the attraction between the two solids (Figure S1(a)) is stronger than the interaction between the air and the solid (Figure S1(b)), then the surface energy is always a positive value.

$\gamma_{12}=W_{121}=W_{212}$

$W_{121}=1 / 2\left(\right.$ breaking attractive energy $\left._{11}\right)+1 / 2\left(\right.$ breaking attractive energy $\left.y_{22}\right)+$

$+\left(\right.$ establishing attractive energy $\left.{ }_{12}\right)$

Where the factor $1 / 2$ is because we only use one of the interfaces when breaking 11 and 22 .

Noting attractive energy as E (namely E11, E22, and E12), we rewrite eq. S2 as: 
$W_{121}=\frac{1}{2}\left(-E_{11}\right)+\frac{1}{2}\left(-E_{22}\right)+\left(E_{12}\right)$

Where the negative sign is for breaking the attractive interaction and positive sign is for establishing it.

Yet, since each attractive energy is defined as a negative property (see section 1 above), then to clarify the signs, we rewrite eq. S3 as follows:

$W_{121}=\frac{1}{2}\left|E_{11}\right|+\frac{1}{2}\left|E_{22}\right|-\left|E_{12}\right|$

Noting that attractive energy is the negative of the work of adhesion, we can also write:

$$
W_{121}=\frac{1}{2} W_{11}+\frac{1}{2} W_{22}-W_{12}
$$

All the equations above are the same, but Eq. S4 and S5 make it obvious that $\mathrm{W}_{121}$ is a positive value when

$\left|E_{11}\right|+\left|E_{22}\right|>2\left|E_{12}\right|$

Inequality S6 is always true because like likes like. This fact is explained well in the book by Jacob Israelachvili ${ }^{1}$. Briefly, considering two body interaction, we can write

$E_{11} \sim d_{1} \times d_{1}=d_{1}^{2}$

and

$E_{22} \sim d_{2} \times d_{2}=d_{2}^{2}$

and

$E_{12} \sim d_{1} \times d_{2}$

where $d_{1}$ and $d_{2}$ represent the dipoles of molecules 1 and 2 .

Now, since $\left(d_{1}^{2}-d_{2}^{2}\right)>0$ then $d_{1}^{2}+d_{2}^{2}>2 d_{1} \times d_{2}$ and therefore

$\gamma_{12}=W_{121}=\frac{1}{2}\left|E_{11}\right|+\frac{1}{2}\left|E_{22}\right|-\left|E_{12}\right|>0$

We conclude sections 1 and 2 by writing that attractive energy is always negative and surface energy is always positive. We proceed to show that, despite of these differences, the maxima and minima of the two coincide.

\section{The maxima and minima of surface energy coincides with that of attractive energy}

Eq. $\mathrm{S} 10$ relates the surface energy, $\gamma_{12}$, to the attraction energy between phases 1 and $2, E_{12}$. We note that $E_{12}$ is always negative and $\gamma_{12}$ is always positive. In addition, we note that $\left|E_{11}\right|$ and $\left|E_{22}\right|$ represent $W_{11}$ and $W_{22}$, namely they represent the work invested in separating phase 1 from phase 1 in vacuum and separating phase 2 from phase 2 in vacuum. Thus, these two values are independent of the conformational contact of phase 1 with phase 2 . The only term in eq. S10 that depends on the contact of phases 1 and 2 is $E_{12}$. Now, $E_{12}$ is a negative number and its contribution to eq $S 8$ is also negative $\left(-\left|E_{12}\right|\right)$. Therefore, the variation of $\gamma_{12}$ coincides with the variation of $E_{12}$. Schematically, the two properties fall on the same curve but with respect to different zero, as shown schematically in Figure 5 of the main text. 


\section{Stages of Motion - Different Solid-Liquid Systems}

\section{A) Pendant DI $\mathrm{H}_{2} \mathrm{O}$ on Silanized (C18) Silicon}

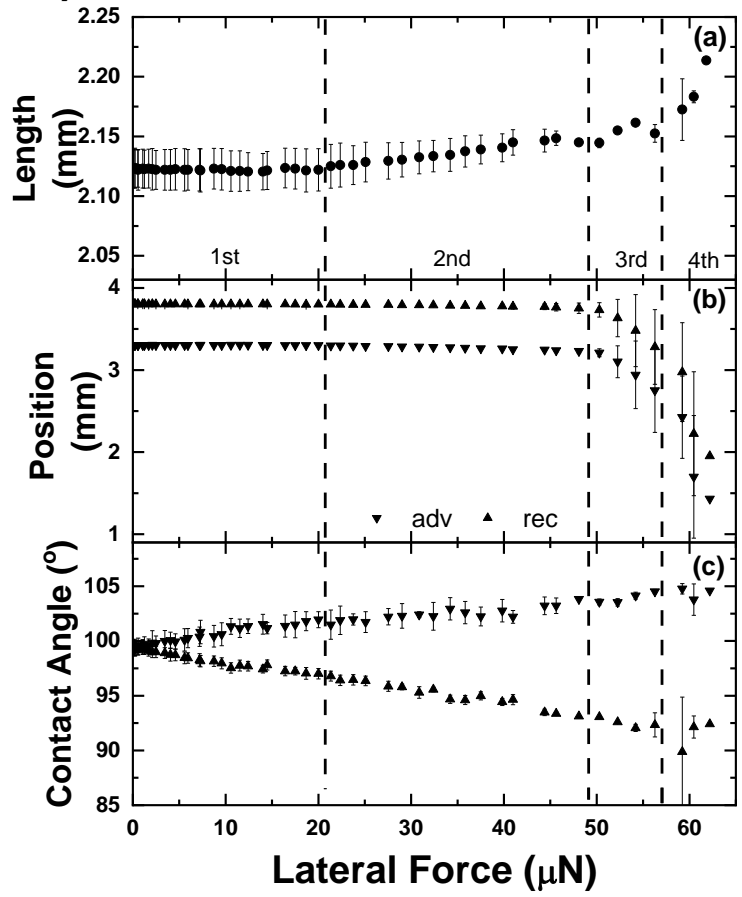

\section{B) Hexadecane on PFOCTS-PDMS}

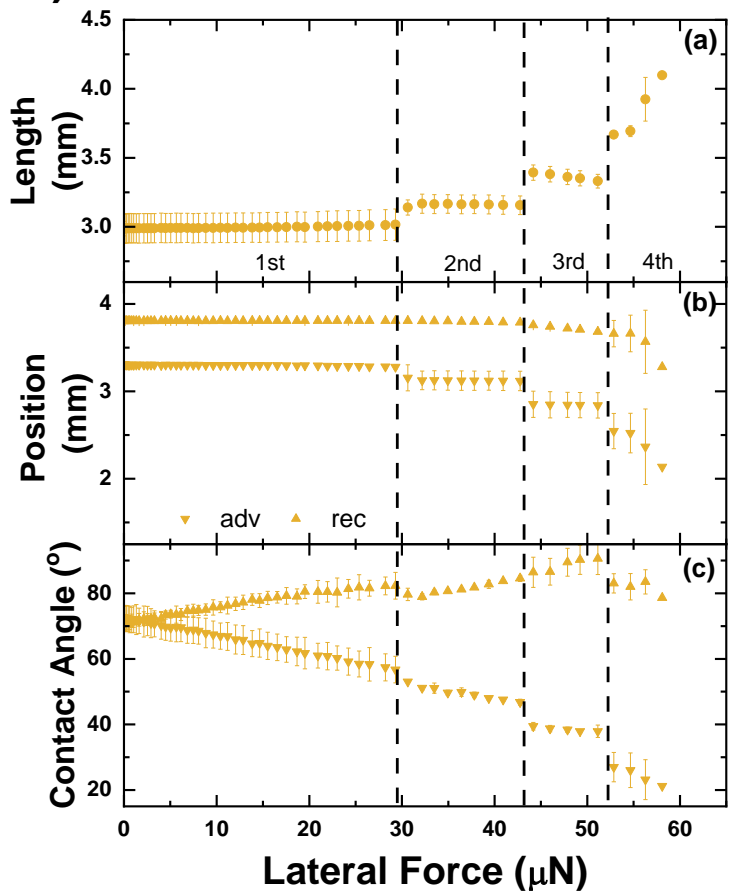

Figure S2: Plots that represent averages of three runs of (a) Drop's length, (b) positions and (c) contact angles for three runs as a function of the lateral force exerted on a A) $3 \mu \mathrm{L}$ water drop (pendant) sliding on a C18 silanized silicon surface and B) $3 \mu \mathrm{L}$ Hexadecane drop sliding on a PFOCTS-PDMS surface.

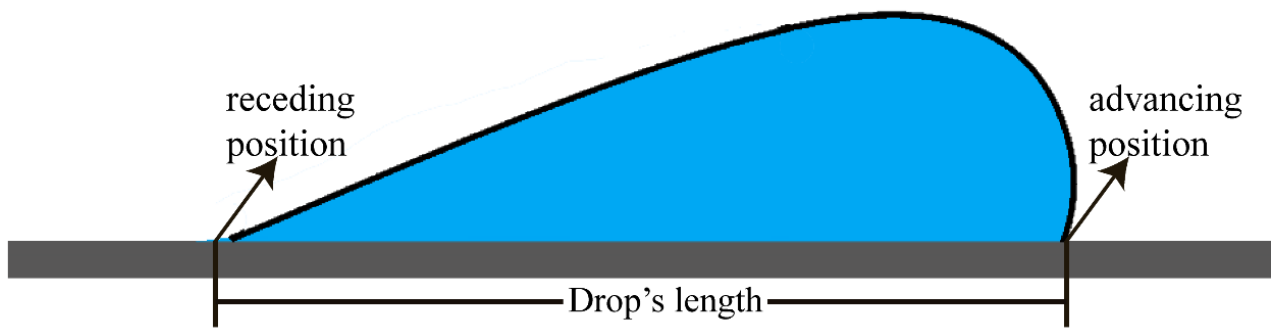

Figure S3: Schematic description of different parameters that were in figures that were used in the main text.

\section{References}

(1) Israelachvili, J. N. Intermolecular and Surface Forces: Third Edition; 2011. https://doi.org/10.1016/C2011-0-05119-0. 